\title{
Fecal microbiota characteristics of Chinese patients with primary lgA nephropathy: a cross-sectional study
}

\author{
Xiaofang $\mathrm{Hu}^{1,2}$, Jie Du², Yuhong Xie ${ }^{1,2}$, Qiong Huang ${ }^{1,2}$, Yi Xiao ${ }^{1,2}$, Juan Chen ${ }^{3}$, Siyuan Yan ${ }^{3}$, \\ Zhicheng Gong ${ }^{1,2^{*}}$ and Shaxi Ouyang ${ }^{3^{*}}$
}

\begin{abstract}
Background: Growing evidence has shown that the gut-renal connection and gut microbiota dysbiosis play a critical role in immunoglobulin A nephropathy (IgAN). However, the fecal microbiome profile in Chinese patients with IgAN remains unknown. A cross-sectional study was designed for the first time to investigate the fecal microbiota compositions in patients with primary IgAN in China and to evaluate the relationship between the fecal microbiome and IgAN clinical presentation.

Methods: Fecal samples were collected from 17 IgAN patients and 18 age-, sex-, and body mass index-matched healthy controls, and bacterial DNA was extracted for $16 \mathrm{~S}$ ribosomal RNA gene sequencing targeting the V3-V4 region.

Results: Fecal samples from the IgAN patients and healthy controls showed differences in gut microbiota community richness and compositions. Compared to the healthy controls, IgAN patients at the phylum level had an increased abundance of Fusobacteria, but a decreased abundance of Synergistetes. The significantly increased genera in the IgAN group were Escherichia-Shigella, Hungatella, and Eggerthella, all of which possess pathogenic potential. Furthermore, the genus Escherichia-Shigella was negatively associated with the estimated glomerular filtration rate (eGFR) but was positively associated with the urinary albumin-to-creatinine ratio (UACR). However, the genus rectale group was present in the IgAN group with a low abundance and was negatively associated with the UACR. Functional analysis disclosed that infection-related pathways were enriched in the IgAN group.
\end{abstract}

Conclusions: We demonstrate that gut microbiota dysbiosis occurs in patients with IgAN, and that changes in gut bacterial populations are closely related to IgAN clinical features, suggesting that certain specific gut microbiota may be a potential therapeutic target for IgAN.

Keywords: Immunoglobulin A nephropathy, Fecal microbiota, Bacterial diversity, Gut microbiota dysbiosis

\footnotetext{
*Correspondence: gongzhicheng2013@163.com; shaxiouyang@163.com

'Department of Pharmacy, Xiangya Hospital, Central South University, No. 87

Xiangya Road, Kaifu District, Changsha 410008, Hunan, China

${ }^{3}$ Department of Nephrology, Hunan Provincial People's Hospital, The

first-affiliated hospital of Hunan normal university, No. 61 Jie-fang West Road,

Fu-Rong District, Changsha 410005, Hunan, China

Full list of author information is available at the end of the article
}

(c) The Author(s). 2020 Open Access This article is licensed under a Creative Commons Attribution 4.0 International License, which permits use, sharing, adaptation, distribution and reproduction in any medium or format, as long as you give appropriate credit to the original author(s) and the source, provide a link to the Creative Commons licence, and indicate if changes were made. The images or other third party material in this article are included in the article's Creative Commons licence, unless indicated otherwise in a credit line to the material. If material is not included in the article's Creative Commons licence and your intended use is not permitted by statutory regulation or exceeds the permitted use, you will need to obtain permission directly from the copyright holder. To view a copy of this licence, visit http://creativecommons.org/licenses/by/4.0/. The Creative Commons Public Domain Dedication waiver (http://creativecommons.org/publicdomain/zero/1.0/) applies to the data made available in this article, unless otherwise stated in a credit line to the data. 


\section{Background}

Currently, immunoglobulin A nephropathy (IgAN) is the most common type of primary glomerular disease in adults worldwide [1, 2], accounting for approximately $58.2 \%$ of primary glomerular diseases in China [3]. The diagnosis of IgAN depends predominately on renal biopsy [4] to detect the deposition of glomerular mesangial immune complexes, which lead to inflammation and result in kidney failure [5]. IgAN has a wide spectrum of clinical features. While some IgAN patients develop the disease rapidly without noticeable symptoms [6], approximately $30-40 \%$ of the cases progress to end-stage renal disease (ESRD) after 20 to 30 years [7]. Although the overproduction of aberrantly glycosylated IgA1 [1] is regarded as an indispensable pathogenic contributor in IgAN, the exact pathogenic mechanism of IgAN is still poorly understood. As the dominant antibody isotype found in mucosal secretions, IgA plays a crucial role in controlling the composition of the gut microbiota by promoting symbiosis among bacteria [8], and secretory IgA has evolved to maintain diverse but stable gut microbial communities [9]. Mucosal abnormalities may be involved in the production of galactose-deficient IgA1, macromolecular immune complexes, and mesangial deposits [10]. Nevertheless, growing evidence has shown that the gut-renal connection and gut microbiota dysbiosis play a critical role in IgAN.

A gut-kidney axis has been implicated in causing chronic kidney diseases, in which a destroyed intestinal mucosal barrier facilitates bacterial lipopolysaccharide (LPS) entry into the circulation, resulting in uremic toxicity and systemic inflammation [11]. In regard to the importance of the gut-renal connection in IgAN [10], a subpopulation of $\gamma \delta \mathrm{T}$ cells that represent the majority of $\gamma \delta \mathrm{T}$ cells in normal gut mucosa has shown a significant decrease in the intestine of patients with IgAN [12]. Additionally, IgAN patients have high levels of serum B-cell activating factor (BAFF) and a proliferation-inducing ligand (APRIL), both of which are associated with the maintenance of tolerogenic immune responses to the microbiota $[13,14]$. BAFF-overexpressing transgenic mice have mesangial deposits of IgA along with high circulating levels of aberrantly glycosylated polymeric IgA, which are associated with IgA-related renal diseases [13]. A recent genome-wide association study has revealed that most IgAN-related loci are associated with immune-mediated inflammatory bowel disease, intestinal barrier maintenance, and response to gut pathogens [15]. Collectively, cumulative evidence indicates that the disorder of intestinal mucosal immune response is the culprit behind the development of IgAN. However, intestinal microbes growing on the mucosal surface are in contact with intestinal epithelial cells and play a regulatory role in the intestinal immune system by altering intestinal permeability and interacting with Toll-like receptors (TLRs) expressed on the mucosal surface cells [16]. Thus, the commensal microbes interact with and influence the host's innate and adaptive immune systems. For example, mouse segmented filamentous bacteria promote the accumulation of proinflammatory T helper 1 (Th1) and Th17 cells in the gutassociated lymphoid tissues [17], whereas other microbes, such as Bacteroides fragilis [18] and Faecalibacterium prausnitzii [18], enhance the accrual of $T$ regulatory cells. Additionally, Grosserichter-Wagener et al. have reported that the composition of intestinal bacteria can affect the antibody reaction activity, Th cell subpopulation, and IgA reaction activity [19]. In recent years, fecal microbiota transplantation (FMT) has been proved to effectively rebuild the intestinal microecological balance. A prospective cohort trial to evaluate the safety and efficacy of FMT in IgAN patients is ongoing [20]. Hence, the gut microbiota is critical to maintaining intestinal immune homeostasis, and defective mucosal microenvironments and unbalanced gut microbiota might be important contributing factors toward the pathogenesis of IgAN [10].

The intestinal microbiota dysbiosis associated with IgAN has previously been studied in Italy [21]. However, geographical origin and dietary habits have a greater impact on gut microbial communities than body mass index (BMI) and sex [22]. Thus far, the profile of fecal microbial communities in Chinese IgAN patients remains unclear. Therefore, in the present study, we aimed to provide new clues for the early diagnosis and treatment of IgAN. We comparatively investigated the microbial communities in the feces of Chinese IgAN patients and healthy controls by using $16 \mathrm{~S}$ ribosomal RNA (rRNA) gene sequencing and evaluated the relationship between the gut microbiota and clinical features of IgAN.

\section{Methods \\ Subjects}

A total of 17 hospitalized patients with IgAN in the Hunan Provincial People's Hospital and 18 age-, sex-, and BMI-matched healthy controls were enrolled in this study. All participants, including patients and control individuals, were Hunan Province natives and Han Chinese. Before they were enrolled, both the patients and healthy controls provided written informed consent. All clinical tests and methods including collection of feces were performed in accordance with relevant guidelines and regulations. This study was approved by the Medical Ethics Committee of the Hunan Provincial People's Hospital. Dietary information over the past three months was questioned, such as consumption of refined carbohydrates, meats, eggs, dairy products, vegetables, and fruits. Dietary habits were assessed by frequency: frequently (once every $\leq 3$ days), sometimes (once every 4-7 days), occasionally (once every $8-30$ days), and none (once for $>1$ month). 


\section{Inclusion and exclusion criteria for IgAN}

To avoid the effects of drugs and other hospital factors on intestinal microorganisms, we included only newly diagnosed IgAN patients without any prior treatment and collected inpatient samples before starting any medication. Samples were further screened after the diagnosis of IgAN was confirmed by renal biopsy. Thus, these patients did not take medications before sample collection. Renal biopsy specimens were evaluated by the presence of four histological features: mesangial hypercellularity $(\mathrm{M})$, endocapillary hypercellularity (E), segmental glomerulosclerosis (S), and tubular atrophy/interstitial fibrosis $(\mathrm{T})$, as defined in the Oxford-MEST classification. The exclusion criteria were as follows: secondary IgAN, such as lupus nephritis, hepatitis B-associated nephritis, and anaphylactoid purpura nephritis; IgAN patients using hormones, angiotensin-convertingenzyme inhibitors/angiotensin II receptor blockers or immunosuppressants; individuals who took antibiotics, vitamins, probiotics, prebiotics, and laxatives within three months prior to sample collection; individuals with a history of cholecystectomy, colectomy or other intestinal diseases; and individuals with diarrhea, constipation, serious infections and complications.

In addition, age-, sex-, and BMI-matched healthy individuals were recruited as controls. These volunteers came to our hospital for a routine medical examination, which confirmed that they were in healthy condition and not taking medications. As for the family members of the patients, since most of them did not have a medical examination and were experiencing a long-term anxiety about the patients' suffering, it was unacceptable to select patients' family members as the control individuals.

\section{Sample size and power analysis}

Sample size and powder analysis were calculated with the help of the G-Power software V3.1.9.4 [23, 24]. The threshold parameter was set by default to type I error of 0.05 and a potency of $80 \%$ and effect size of 0.5623 , and we calculated that a minimum of 15 samples were required. Effect size was obtained according to preliminary experimental results and demonstrated that the sample sizes we used were adequate to address our research questions.

\section{Clinical data and biochemical analysis}

Personal information, including sex, age, weight, and height, was collected, and BMI was calculated. Fasting peripheral venous blood samples were drawn in the morning, and serum biochemical parameters were analyzed by using automatic biochemical analyzers (Beckman Coulter AU5800, Brea, CA, USA), including blood urea nitrogen $(\mathrm{BUN})$, serum creatinine $(\mathrm{sCr})$, cystatin $\mathrm{C}$ $(\mathrm{CysC})$, fasting blood glucose (FBG), total cholesterol (TC), and triglycerides (TG). On the first morning, urine samples were collected and centrifuged; then,urinary albumin (uALB) was quantified by a turbidimetric inhibition immune assay, urine creatinine $(\mathrm{uCr})$ was determined with a sarcosine oxidase assay, C-reactive protein (CRP) was quantified by immunoturbidimetric assay and the estimated glomerular filtration rate (eGFR) was calculated using the Chronic Kidney Disease Epidemiology Collaboration (CKD-EPI) equation [25].

\section{Fecal sample collection and DNA extraction}

Fecal samples were collected using sterile harvesters and frozen in sterile cryotubes at $-80^{\circ} \mathrm{C}$ in no more than 30 min. Bacterial DNA was extracted from the fecal samples using the HiPure Stool DNA Kit (Magen, Guangzhou, China) according to the manufacturer' instructions.

\section{PCR amplification and amplicon sequencing}

The V3-V4 region of the 16S rDNA gene from the fecal DNA was amplified using the barcoded primers $341 \mathrm{~F}$ (CCTACGGGNGGCWGCAG) and 806R (GGACTACH VGGGTATCTAAT). PCR amplification was carried out in a $50 \mu \mathrm{L}$ reaction system containing $5 \mu \mathrm{L}$ of $10 \times \mathrm{KOD}$ buffer, $5 \mu \mathrm{L}$ of $2.5 \mathrm{mM}$ dNTPs, $1.5 \mu \mathrm{L}$ of $5 \mu \mathrm{M}$ primers, $1 \mu \mathrm{L}$ of KOD polymerase, and $100 \mathrm{ng}$ of template DNA. PCRs were performed using the following cycle conditions: predenaturation at $95^{\circ} \mathrm{C}$ for $2 \mathrm{~min}$, followed by 27 cycles of denaturation at $98^{\circ} \mathrm{C}$ for $10 \mathrm{~s}$, annealing at $62{ }^{\circ} \mathrm{C}$ for $30 \mathrm{~s}$, extension at $68^{\circ} \mathrm{C}$ for $30 \mathrm{~s}$, and a final extension at $68^{\circ} \mathrm{C}$ for $10 \mathrm{~min}$. The amplified products were purified using the AxyPrep DNA Gel Extraction Kit (Axygen Biosciences, Union City, CA, USA) and then sequenced on the Illumina HiSeq 2500 platform by Gene Denovo Biotechnology. (Guangzhou, China).

\section{Data processing and analysis}

The raw data was subjected to vigorous quality control, tag splicing, tag filtering, tag dechimerization, and deredundancy processing to obtain effective sequence tags; Uparse software was used to cluster all effective tags of the samples to the same operational taxonomic units (OTUs) based on amplicon sequence similarity $\geq 97 \%$. The most abundant sequence from each OTU was selected as the representative sequence for species annotations by using the Ribosomal Database Project (RDP) Naive Bayes classifier. After annotation, all OTUs $<10$ or proportion $<1 \%$ were discarded in the diversity analysis progress. In addition, $\alpha$ and $\beta$ diversity of microbial communities was analyzed using the standard Quantitative Insights into Microbial Ecology (QIIME) software. For $\beta$ diversity analysis, the Weighted UniFrac based on phylogenetic distances was used to assess the similarities between a pair of samples. Principal coordinate analysis ( $\mathrm{PCoA})$ was performed by multivariate analyses to compare the differences between the microbial community distances of the samples. Functional analyses of microbial communities were performed using the software Tax4Fun. SPSS (ver. 
Table 1 Clinical and demographic features of IgAN patients and healthy controls

\begin{tabular}{llll}
\hline & Healthy group $(n=18)$ & IgAN group $(n=17)$ & $P$-value \\
\hline Sex (Female/Male) & $4 / 14$ & $3 / 14$ & 0.735 \\
Age (years) & $49.72 \pm 8.39$ & $44.76 \pm 10.53$ & 0.132 \\
BMI $\left(\mathrm{kg} / \mathrm{m}^{2}\right)$ & $23.16 \pm 1.39$ & $22.18 \pm 2.09$ & 0.108 \\
CysC $(\mathrm{mg} / \mathrm{L})$ & $0.55 \pm 0.08$ & $0.82 \pm 0.43$ & 0.011 \\
BUN $(\mathrm{mmol} / \mathrm{L})$ & $4.88 \pm 0.99$ & $5.64 \pm 2.29$ & 0.208 \\
Scr $(\mu \mathrm{mol} / \mathrm{L})$ & $56.35 \pm 8.65$ & $101.43 \pm 87.04$ & 0.036 \\
eGFR $\left(\mathrm{ml} / \mathrm{min} / 1.73 \mathrm{~m}^{2}\right)$ & $107.13 \pm 12.52$ & $83.16 \pm 32.49$ & 0.006 \\
uACR $(\mathrm{mg} / \mathrm{g})$ & $9.61 \pm 3.00$ & $949.49 \pm 814.11$ & 0.000 \\
GLU $(\mathrm{mmol} / \mathrm{L})$ & $5.03 \pm 0.54$ & $5.08 \pm 0.51$ & 0.764 \\
TC $(\mathrm{mmol} / \mathrm{L})$ & $4.43 \pm 0.71$ & $4.84 \pm 0.99$ & 0.160 \\
TG $(\mathrm{mmol} / \mathrm{L})$ & $1.29 \pm 0.23$ & $1.54 \pm 0.62$ & 0.119 \\
CRP & $0.47 \pm 0.29$ & $1.12 \pm 1.17$ & 0.030
\end{tabular}

Frequency of pathologic features (percentages) in 17 biopsies scored according to Oxford Classification

mesangial hypercellularity $(\mathrm{M}) \mathrm{M0}=12 \% \mathrm{M} 1=88 \%$

endocapillary hypercellularity (E) E0 $=88 \% \mathrm{E} 1=12 \%$

segmental glomerulosclerosis (S) S0 $=71 \%$ S1 $=29 \%$

tubular atrophy/interstitial fibrosis (T) T0 $=76 \% \mathrm{~T} 1=18 \% \mathrm{~T} 2=6 \%$

Note: Results are expressed as the mean \pm SD and ratio. Abbreviations: $S D$ standard deviation; $B M I$ body mass index; $C y s C$ Cystatin $C ; B U N$ blood urea nitrogen; $C r$ creatinine; eGFR estimated glomerular filtration rate; $U A C R$ urinary albumin-to-creatinine ratio; GLU glucose; TC total cholesterol; TG Triglyceride; CRP C-reactive protein

22.0) and R software (ver. 3.1.0) were used for the statistical analysis. Comparisons between continuous variables were performed using the Student $t$ test. Wilcoxon rank sum test was used to compare the flora abundance of the two groups. The association between categorical variables was investigated by Chi-square or Fisher exact tests. Spearman correlation analysis was used to evaluate the correlation between fecal microbiota and IgAN clinical parameters, and random forest (RF) model was used to evaluate the disease status based on microbiota profiles (significantly different taxa at genus level from Wilcoxon rank sum test). $P<0.05$ was considered statistically significant.

\section{Experimental validation in public database}

Gut microbiota data associated with all kidney diseases were downloaded from database of human gut

Table 2 Frequency list of dietary habits in IgAN patients and healthy controls

\begin{tabular}{llll}
\hline & $\begin{array}{l}\text { Healthy group } \\
(\mathrm{F} / \mathrm{S} / \mathrm{O} / \mathrm{N})\end{array}$ & $\begin{array}{l}\text { IgAN group } \\
(\mathrm{F} / \mathrm{S} / \mathrm{O} / \mathrm{N})\end{array}$ & $\boldsymbol{P}$-value \\
\hline Refined carbohydrates & $13 / 3 / 2 / 0$ & $12 / 4 / 1 / 0$ & 0.783 \\
Meats and Eggs & $10 / 6 / 2 / 0$ & $11 / 4 / 2 / 0$ & 0.811 \\
Dairy products & $8 / 6 / 2 / 2$ & $7 / 5 / 2 / 3$ & 0.954 \\
Vegetables and fruits & $14 / 3 / 1 / 0$ & $13 / 2 / 2 / 0$ & 0.763 \\
intotal & 18 & 17 & \\
\hline
\end{tabular}

Note: $F$ frequently; $S$ sometimes; $O$ occasionally; $N$ none; microbiota (https://gmrepo.humangut.info/home) and employed as reference data. Up to december 2019, a total of 163 samples associated with kidney diseases were downloaded from the database of human gut microbiota. Only those gut microbiota which were found in $\geq$ two samples and with median relative abundances $\geq 0.01 \%$ were taken into consideration. The relative abundances of all gut microbes (OTUs) downloaded from the database of human gut microbiota were calculated by $\mathrm{R}$ software. All annotated results (OTUs) in our study were compared to the public data.

\section{Results}

Basic clinical and biochemical characteristics of IgAN patients and healthy controls

The individual demographics, biochemical characteristics, eating habits, and pathological features of the studied subjects are summarized in Table 1 . There were no significant differences between the IgAN patients and healthy controls in age, sex, and BMI as well as the BUN, glucose, TC, and TG levels. However, serum CysC and $\mathrm{sCr}$, the urinary albumin-to-creatinine ratio (uACR), and blood CRP were significantly increased, but eGFR was significantly reduced in the IgAN patients when compared to the healthy controls. Moreover, there were no significant differences in dietary habits between the two groups (Table 2). 


\section{$a$ and $\beta$ diversity between IgAN patients and healthy controls}

To contrast the microbial community richness and composition between the IgAN patients and healthy controls, we evaluated the $\alpha$ and $\beta$ diversity of fecal microbiota. With the exception of the Shannon diversity index, other $\alpha$ diversity indexes, including Chao, ACE, and observed species diversity, were significantly decreased in the IgAN patients compared to those in the healthy controls (Fig. 1a), indicating that the microbial community richness of the gut microbiota is significantly lower in the IgAN patients than in the healthy controls. Based on the PCoA plot, we found a separation trend in the $\beta$ diversity between the IgAN patients and healthy controls (Fig. 1b). Also, a significant difference in $\beta$-diversity was observed between the two groups based on the weighted UniFrac clustering (ANOSIM $\mathrm{R}=$ $0.076, P=0.035$; Fig. 1c), suggesting that the fecal microbial community structure in the IgAN patients is significantly different from that in the healthy controls according to the presence of fecal OTUs.
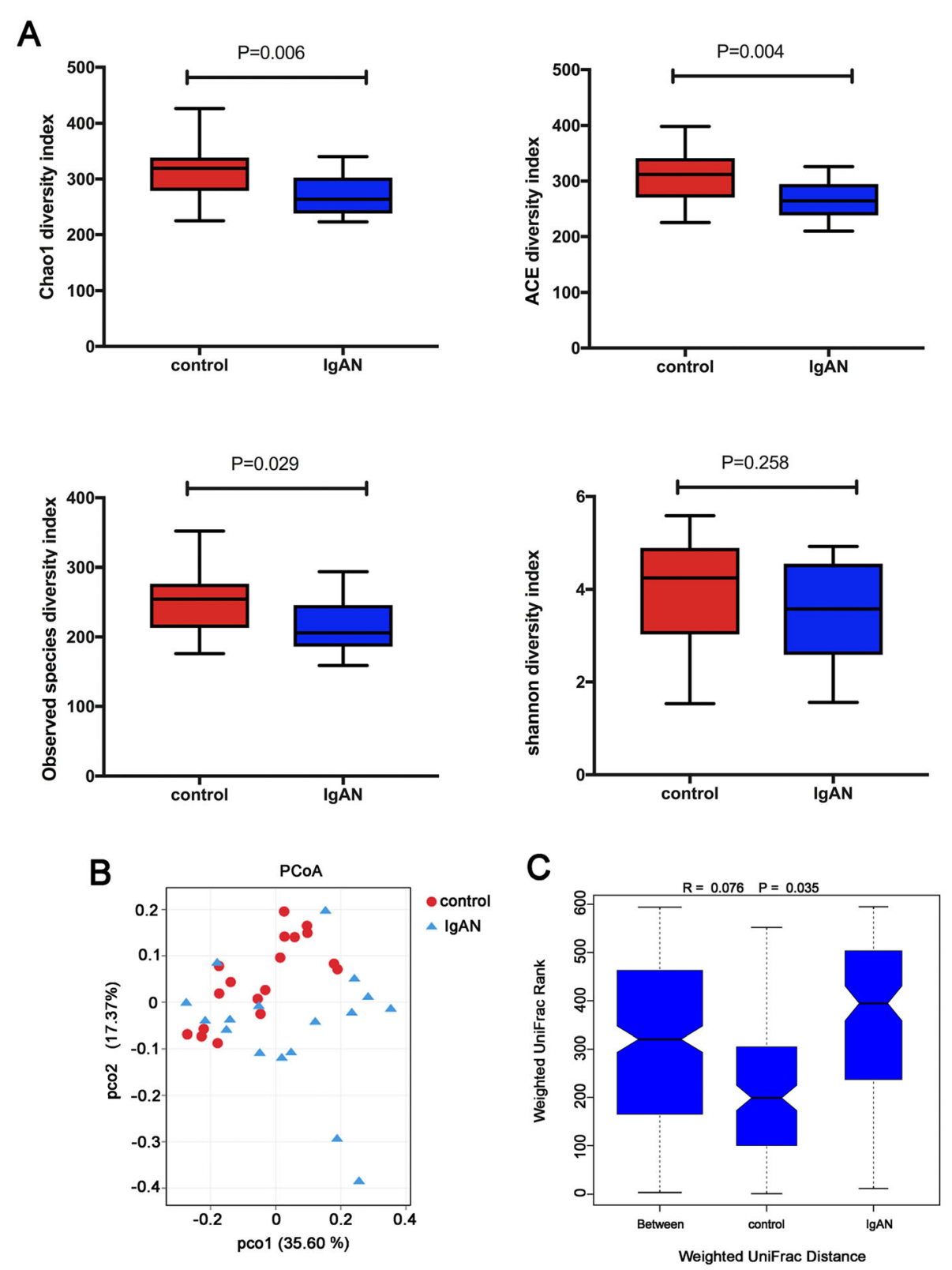

Fig. 1 Comparison of diversity indexes between the IgAN patients and healthy controls. (a) The differences of a-diversity indexes (Chao1, ACE, Observed species, and Shannon index) between the IgAN patients and healthy controls. (b) Principal coordinates analysis (PCoA) with weighted UniFrac distance for bacterial communities between the IgAN patients and healthy controls. (c) Analysis of similarities (ANOSIM) based on the weighted UniFrac distance between the IgAN patients and healthy controls 


\section{Bacterial taxa differences between IgAN patients and healthy controls}

Both IgAN patients and healthy controls had typical microbiome structures. Most bacteria fell within the phyla Bacteroidetes, Firmicutes, Proteobacteria, and Verrucomicrobia (Fig. 2a). There was no statistically significant difference in the microbiome structure between the two groups, although the IgAN patients showed an increased abundance of Firmicutes and a decreased abundance of Bacteroidetes and Proteobacteria. Note that compared to the healthy controls, the IgAN patients showed an increased abundance of Fusobacteria but a decreased abundance of Synergistes $(P<$ 0.05). Within Proteobacteria, the Enterobacteriaceae abundance was significantly higher in the IgAN patients than in the healthy controls at the family level $(P<0.05)$. For the Synergistetes family, a significant decrease was found in the Synergistaceae abundance in the IgAN group compared to that in the healthy group $(P<0.05)$ (Table 3$)$.

At the genus level, Bacteroides, Faecalibacterium, Prevotella-9, Escherichia-Shigella, and Megamonas were the most abundant genera in both groups. Some of these genera showed a significant difference between these two groups (Fig. 2b). The abundances of the genera Escherichia-Shigella,
Hungatella, and Eggerthella were higher in the IgAN group than in the healthy group, whereas the abundances of the genera rectale group, Barnesiella, Ruminococcaceae NK4A214_group, Prevotellaceae_NK3B31_group, Prevotellaceae_UCG-001 Coprococcus_2, Lachnospiraceae_FCSO2O_ group, and Pyramidobacter were higher in the healthy group than in the IgAN group $(P<0.05)$ (Table 3$)$. These data suggest that the taxonomic abundances of microbial communities are different in the IgAN patients and the healthy controls.

\section{Random forest (RF) predictive modeling}

To evaluate the disease status of the IgAN patients based on an ensemble of decision trees, we used RF to build a predictive model according to the fecal microbiota profiles with the significantly different taxa abundances at the genus level from Wilcoxon rank-sum test as the input. Among the fecal microbiota, 7 genera predicted IgAN with a sensitivity of $82.4 \%$ and a specificity of 72.2\% (AUC $=0.833,95 \%$ CI: 0.699-0.967) using the RF model (Fig. 2c and d), suggesting that certain genera of fecal microbiota are predictive of the IgAN disease state.
A

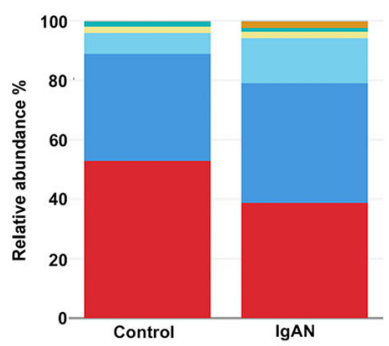

C

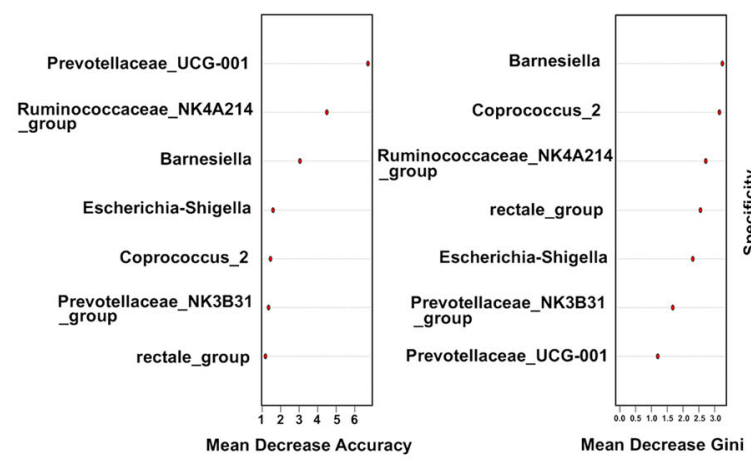

B

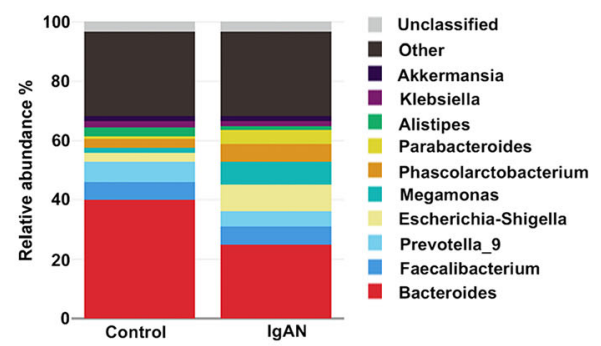

D

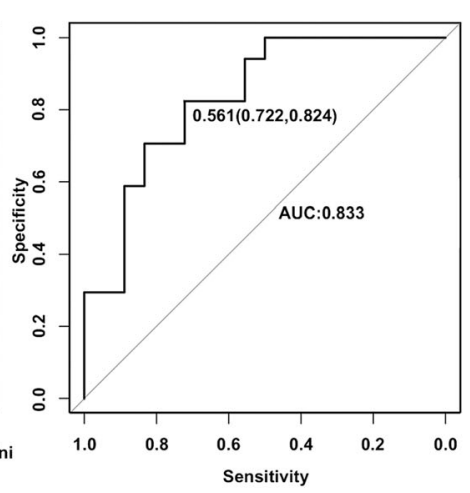

Fig. 2 Relative abundance and taxonomic differences of fecal microbiota in the IgAN patients and healthy controls. (a) Microbiome composition in the two groups at the phylum level. (b) Microbiome composition in the two groups at the genus level. The figure shows the top 10 species in each group based on their relative abundances. (c) The predictive model based on the relative abundances at genus level by using a RF model. The importance of each genus in the predictive model was evaluated by the mean decreasing accuracy and the Gini coefficient. (d) ROC curve analysis generated by the RF using 7 genera in the fecal microbiota 
Table 3 Relative abundance of fecal microbiota in IgAN patients and healthy controls

\begin{tabular}{|c|c|c|c|}
\hline & $\begin{array}{l}\text { Healthy } \\
\text { group }\end{array}$ & $\begin{array}{l}\text { IgAN } \\
\text { group }\end{array}$ & $\boldsymbol{P}$-value \\
\hline \multicolumn{4}{|l|}{ Phylum level } \\
\hline p-Fusobacteria & 0.2269 & 2.2622 & 0.031 \\
\hline p-Synergistetes & 0.0297 & 0.0097 & 0.026 \\
\hline \multicolumn{4}{|l|}{ Family level } \\
\hline f-Enterobacteriaceae & 5.9760 & 12.4897 & 0.031 \\
\hline f-Synergistaceae & 0.0297 & 0.0097 & 0.026 \\
\hline \multicolumn{4}{|l|}{ Genus level } \\
\hline g-Escherichia-Shigella & 3.0358 & 8.8629 & 0.048 \\
\hline g-rectale_group & 2.5503 & 0.9346 & 0.018 \\
\hline g-Barnesiella & 0.5212 & 0.2121 & 0.021 \\
\hline $\begin{array}{l}\text { g- Ruminococcaceae_NK4A214_ } \\
\text { group }\end{array}$ & 0.3045 & 0.0806 & 0.024 \\
\hline g-Prevotellaceae_NK3B31_group & 0.1767 & 0.1188 & 0.011 \\
\hline g-Prevotellaceae_UCG-001 & 0.2161 & $7.059 \mathrm{e}-05$ & 0.003 \\
\hline g-Coprococcus_2 & 0.1134 & 0.05403 & 0.007 \\
\hline g- Hungatella & 0.0062 & 0.1444 & 0.026 \\
\hline g-Rikenellaceae_RC9_gut_group & 0.1037 & 0 & 0.002 \\
\hline $\begin{array}{l}\text { g- Lachnospiraceae_FCSO2O_ } \\
\text { group }\end{array}$ & 0.0414 & 0.0187 & 0.014 \\
\hline g- Eggerthella & 0.0156 & 0.0417 & 0.001 \\
\hline g-Pyramidobacter & 0.0293 & 0.0089 & 0.004 \\
\hline
\end{tabular}

\section{Association between fecal microbiota and IgAN clinical characteristics}

We evaluated the correlation between fecal microbiota (at the phylum level and genus level) and IgAN clinical parameters (Fig. 3) and found that the phylum Proteobacteria and the genera Escherichia-Shigella, Sneathia, and Plesiomonas were negatively associated with the eGFR, while the genus Blautia was positively associated with the eGFR. Moreover, the genera Escherichia-Shigella, Sneathia, and Parabacteroides were positively associated with the $\mathrm{uACR}$, but the genus rectale_group was negatively associated with the uACR. Additionally, the genera Hungatella, Sellimonas, and gnavus_group were positively associated with CRP. These findings suggest a strong correlation of altered fecal microbiota with the clinical features of the IgAN patients.

\section{Functional analysis}

Tax4Fun [26] based on the SILVA rRNA database as a reference was used to predict the relative abundances of functional categories from databases such as the Kyoto Encyclopedia of Genes and Genomes (KEGG) ortholog (KO) database. Our results show differential KOs identified between the IgAN and healthy groups $(P<0.05$; Fig. 4a). At levels 2 and 3 of the KEGG pathways, the microbial gene functions, including those pathways involved in infectious diseases, glyoxylate and dicarboxylate metabolism, and Salmonella infection, were higher in the fecal microbiome of the IgAN group, whereas the microbial gene functions related to starch and sucrose metabolism, N-glycan biosynthesis, carbohydrate digestion and absorption, and protein export were higher in the fecal microbiome of the healthy group $(P<0.05$; Fig. $4 b)$, indicating the differential function of the fecal microbiota between diseased and healthy individuals.

\section{In comparison to public database}

Of all tested genera, 101 microbial genera including Escherichia-Shigella, Bacteroides, Klebsiella, Fusobacterium were found in both our study and the pubic database, and 79 microbial genera unique to our study were also found (Supplementary materials 1 ). In addition, The significantly differential genera between disease and healthy groups from the database of human gut microbiota and our sequencing data were compared. The abundance of the genus Escherichia-Shigella was higher in the disease group, and the abundances of the genera Barnesiella and Pyramidobacter were lower in the disease group. Unfortunately, the genus Eggerthella was found decreased in the database of human gut microbiota for kidney diseases, but increased in our study.
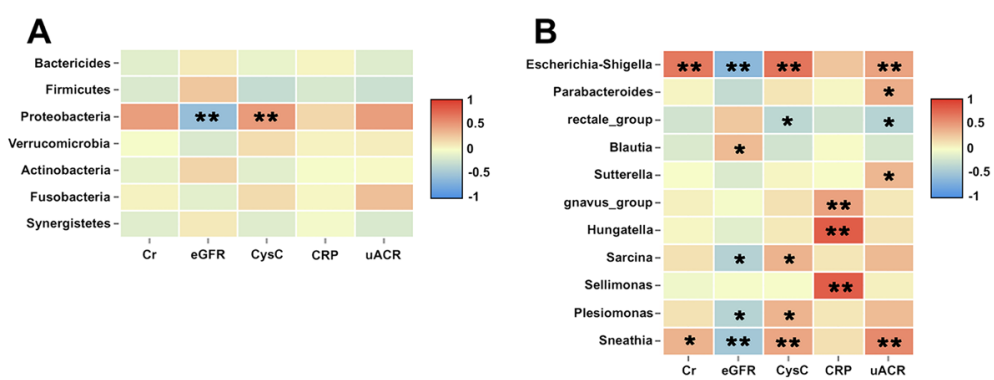

Fig. 3 Heatmaps showing correlations between fecal microbiota and IgAN clinical parameters. (a) Correlations between microbiota phyla and IgAN clinical characteristics. (b) Correlations between microbiota genera and IgAN clinical characteristics. The intensity of the color indicates the $r$ value (correlation). The red color represent positive score and the blue color represent negative score. ${ }^{*} P<0.05$ and ${ }^{* *} P<0.01$ 

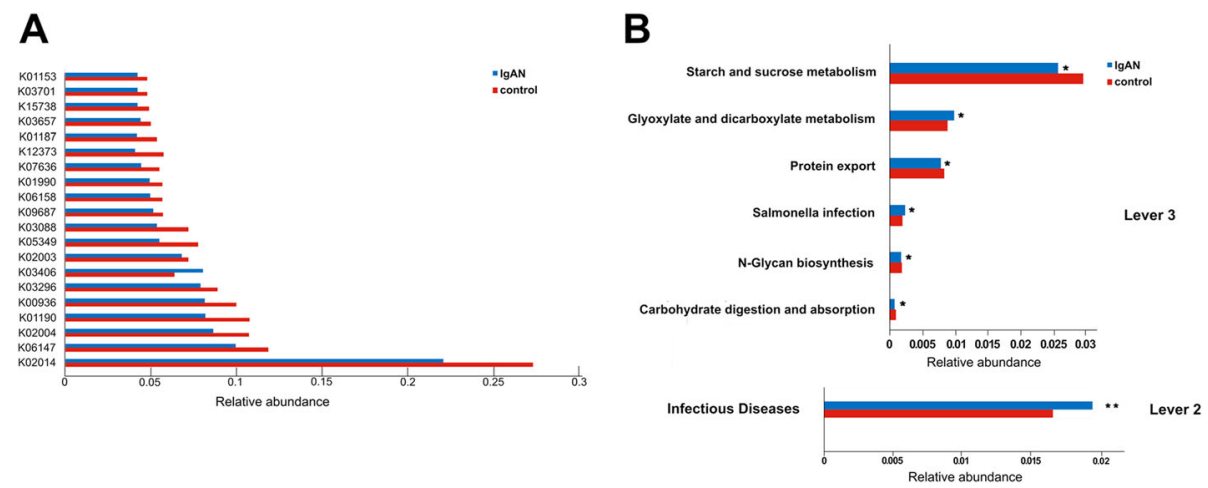

Fig. 4 Predicted microbial functions using Tax4fun. (a) The top $20 \mathrm{KO}$ s with significantly different abundances between the IgAN patients and healthy controls. (b) Significant KEGG pathways at level 2 and 3 between the IgAN patients and healthy controls. ${ }^{*} P<0.05$ and ${ }^{* *} P<0.01$

The genera Hungatella, rectale_group, Coprococcus_2 and Lachnospiraceae_FCSO20_group that were significantly different in our study were not found in the database (no relative abundance results were annotated). These results suggest that all patients with kidney diseases may share certain core microorganisms regardless of the impact of the patients' survival environment and etiology, while some bacterial taxa may be related to the pathological characteristics of IgA nephropathy.

\section{Discussion}

Previous studies have reported that patients with celiac disease and inflammatory bowel disease are more likely to develop IgAN [27-29]. The immune system responds vigorously to microbial infection while permitting lifelong colonization by the microbiome. The microbiome could mediate stable colonization of the gut through coopting IgA responses [30]. Differences in the gut microbial composition and diversity have been observed in Italian Caucasians with IgAN and are closely associated with clinical phenotype [21]. Here, we determined for the first time the fecal microbial composition in Chinese patients with primary IgAN. Our results demonstrated that the community richness of fecal microbiota in the IgAN patients was significantly lower than that in the healthy controls, in agreement with another observation [21]. Note that we confirmed a significant difference in the $\beta$-diversity indexes between the diseased and healthy subjects, which has not been previously reported. These findings indicate that fecal microbiota dysbiosis occurs in Chinese patients with primary IgAN. Taxonomic differences in fecal microbiota between Italian patients and Chinese patients may be due to distinct cultural backgrounds, geographical locations, and eating habits. It has been reported that there are significant differences in the intestinal bacterial diversity among populations of different cultures and geographical regions [31], and dietary factors largely affect microbial composition and function [32]. Therefore, it is important to explore the etiology and therapeutic approach of intestinal flora in the Chinese population.

We found that at the phylum level, Bacteroidetes, Firmicutes, and Proteobacteria were the dominant bacteria in all tested samples. This observation is in agreement with a previous report that patients with nonprogressive or advanced IgAN had a low abundance of Bacteroidetes but a high abundance of Firmicutes [21]. We also found that the abundance of Synergistetes, mainly related to the decreased abundance of the genus Pyramidobacter, was significantly decreased in IgAN patients. As ruminal microbes, Pyramidobacter is believed to participate in the synthesis of thiamine [33, 34], which is important for the maintenance of gut-associated lymphoid tissues [35, 36]. Therefore, it is reasonable to speculate that Pyramidobacter may provide some health benefits to patients with IgAN. More importantly, IgAN patients had an increased abundance of Fusobacteria, Proteobacteria, Enterobacteriaceae, and the specific genus Escherichia-Shigella, most of which are opportunistic pathogens. Fusobacteria are gram-negative obligate anaerobic nonspore-forming bacilli that represent a small proportion of the microbial community, and clinically invasive Fusobacterial infections are rare [37]. Nevertheless, recent studies have shown that Fusobacteria are associated with Crohn's disease, ulcerative colitis, and colorectal cancer [38]. Moreover, the enrichment of Proteobacteria is considered a potential microbial diagnostic signature of dysbiosis and increases the risk of host diseases [39]. As the most common commensal flora in the host-mediated inflammatory response, the excessive proliferation of Enterobacteriaceae driven by inflammation is closely related to the development of various human diseases [40]. For example, hyperproliferative Enterobacteriaceae is present in the feces of ESRD patients [41], and bacterial DNA from the genera Escherichia and Enterobacter is also detectable in the blood of ESRD patients [42]. We also found that some genera that produce 
short-chain fatty acids (SCFAs), including Ruminococcaceae_NK4A214_group, Coprococcus_2, and Lachnospiraceae_FCSO20_group, became less in the IgAN patients. SCFAs are generally considered to have a number of important roles in maintaining health, such as acting as a special nutrient and energy source for the intestinal epithelium, protecting the intestinal mucosal barrier, reducing inflammation, and enhancing the motility of the gastrointestinal tract [43]. Barnesiella is one of the most abundant genera detected in the mouse intestine, has antiinflammatory protective effects in animal models [44, 45], and was found to be significantly reduced in the IgAN patients in this study. Recent studies observed that the genus Barnesiella was the most abundant novel classification in the healthy human gut microbiome [46] and correlated with a healthy state of subjects [47]. The major end products derived from Barnesiella are butyric and isobutyric acids [48], which are responsible for the beneficial effects of the bacteria. Furthermore, members of the Prevotellaceae possess phosphotransbutyrylase and butyrate kinase, which are key enzymes in the butyrate synthesis pathway [49]. Prevotellaceae_NK3B31_group and Prevotellaceae_ UCG-001 of the Prevotellaceae family were also found to be significantly decreased in the IgAN patients. The reduced abundance of Prevotellaceae has also been reported in the stool of ESRD patients [49] and uremia animals [50]. It is well known that intestinal epithelial cells are the first line of cellular defense against pathogen invasion, and that butyrate serves as the primary energy source for intestinal epithelial cells. Butyrate not only regulates stem cell turnover in the intestinal epithelial crypt, but also enhances the bactericidal function of macrophages by shaping their transcription and metabolism [51]. Collectively, the presence of IgAN is related to the abundant change in some bacterial taxa. Our RF models successfully predicted IgAN using several differential several genera with a high sensitivity and specificity. Spearman correlation analysis confirmed that some opportunistic pathogens, such as Escherichia-Shigella and Sneathia, were positively correlated with the uACR but negatively correlated with the eGFR, both of which are classical renal damage markers. We also found that the genus rectale_group was negatively correlated with the uACR and that the genus Blautia was positively associated with the eGFR. Additionally, by comparing with the database, we found that 101 genera in our study were consistent with the public database, which indirectly validated the accuracy of our results. And some new potential kidney disease-related microorganisms were annotated in our study, but not found in the public database, which may enrich the public data of kidney-related gut microorganisms. At the same time, we also found that the genera Escherichia-Shigella, Barnesiella, and Pyramidobacter showed the same variation characters in known public database and our study, suggesting their potential role as biomarkers for kidney disease. In contract to public database, we found that the abundance of Eggerthella was significantly increased in IgAN patients. The implication of Eggerthella in inflammatory diseases have been reported in numerous studies [52]. For example, the increased abundance of Eggerthella was associated with type II diabetes [53], rheumatoid arthritis [54] and Crohn's disease [55], which maybe support the phenomena of our study. Taken together, these results indicate that changes in the composition of specific bacteria may be helpful for the early diagnosis and prediction of IgAN.

Studies have shown that individuals with a low bacterial richness are characterized by a more inflammatory phenotype [56]. The association between increased Enterobacter and decreased renal function has been confirmed in many studies $[57,58]$. The mechanisms behind these phenomena may be attributable to the inflammatory immune response and intestinal susceptibility of IgAN. For instance, the mucosal infection of IgAN patients triggers hematuria and damages the intestine, resulting in acute diarrhea [10]. IgAN patients usually show increased intestinal permeability [59], which facilitates the absorption and circulation of bacterial LPS derived from the Escherichia genus [60]. Animal studies [61] have demonstrated that the expansion of Proteobacteria (specifically Escherichia-Shigella) and enrichment of genes involved in LPS biosynthesis are parallel to the increased levels of LPS in the feces and circulation. LPS and transmembrane signal transduction holds the key to the inflammatory effects of cells. By analyzing mesangial cell proteomics and glomerular transcriptomics, Liu et al. [62] found that most identified pathways induced in mesangial cells by galactose-deficient IgA are involved in inflammation. A link between LPS exposure and defective IgA galactosylation has been confirmed by a study showing that bacterial LPS activates TLR4 in cultured peripheral B lymphocytes to induce Cosmc expression critical for the activity of the enzyme galactosyltransferase [63]. TLR4, the receptor for LPS from Escherichia coli, has been reported with increased mRNA expression in peripheral lymphomononuclear cells of patients with IgAN and in children with IgA vasculitis; in addition, TLR4 is correlated with the signs of innate immunity activation and proteinuria $[64,65]$. Pathway analyses based on KEGG analysis in our study demonstrated that the gut microbiota in patients with IgAN is enriched in multiple pathways associated with infectious diseases. This finding further supports the concept that systemic immune activation and inflammation triggered by gut microbiota-associated LPS signaling play an important role in the development and progression of IgAN. In addition, it is generally believed that the availability of carbohydrates and nitrogen is the most important determinant of microbial metabolism in the colon. In the absence of carbohydrates, $\alpha$-amino nitrogen can produce potentially toxic end products mainly 
through fermentation. It has been reported that the $\alpha$ amino nitrogen fermentation pattern is the key player in gut ecosystem disorders in CKD patients [66], which may contribute to IgAN.

It should be noted that our study has certain limitations. First, this is a cross-sectional study. To reduce the effects of individual differences, a longitudinal study focusing on microbial differences at the different stages of IgAN is needed. Second, our sample size is relatively small; therefore, a large-scale study involving different populations is needed to confirm our results. Third, although Tax4Fun provides functional information about gut microbiota, its limitation is obvious compared with the information provided by shotgun metagenomics analysis. Nevertheless, we generated solid data supporting the role of altered gut microbiota in the pathogenesis of Chinese IgAN patients in this well-matched (i.e., age, BMI, consistency of medication) cohort with the use of next-generation sequencing of specific genomic regions of the microbiome.

\section{Conclusions}

Overall, we collected evidence for gut microbiota dysbiosis in IgAN patients from the Hunan region of China. Our finding regarding the relation of some fecal microbiota to IgAN clinical characteristics will surely help enhance our understanding of the pathogenesis of IgAN and provide potential novel therapeutic options for patients with IgAN by targeting gut microbiota.

\section{Supplementary information}

Supplementary information accompanies this paper at https://doi.org/10 1186/s12882-020-01741-9.

Additional file 1: Supplementary materials 1. Comparison of all genera between our result and public database.

\section{Abbreviations}

BMI: Body mass index; eGFR: Estimated glomerular filtration rate; ESRD: End stage renal disease; IgAN: Immunoglobulin A nephropathy;

LPS: lipopolysaccharide; RF: Random forest; UACR: Urinary albumin-tocreatinine ratio

\section{Acknowledgements}

Not applicable.

\section{Declarations}

None.

\section{Authors' contributions}

Research idea and study design: XFH, ZCG, JD. Data acquisition: XFH, SXOY, JC, SYY. Data analysis/interpretation: XFH, JD, YHX, QH,YX. Manuscript drafting: $X F H, J D$. Editing and revising: $X F H, J D, S X O Y, Y H X$. All authors have approved the submitted version and agreed to be accountable for the author's own contributions.

\section{Funding}

This work is supported by the National Natural Science Foundation of China (No. 81770739 to Zhicheng Gong, No. 81703592 to Jie Du and No. 81673492 to Qiong Huang). No funders had a role in the design of the study, data collection, analysis and data interpretation, or writing of the manuscript.
Availability of data and materials

The datasets used and analyzed in this study are available from the first author and corresponding author on reasonable request.

Ethics approval and consent to participate

This study was approved by the Medical Ethics Committee of the Hunan Provincial People's Hospital, and based on the principles of the Declaration of Helsinki. Written informed consent was obtained from all participants.

\section{Consent for publication}

Not applicable.

\section{Competing interests}

The authors declare that they have no competing interests.

\section{Author details}

${ }^{1}$ Department of Pharmacy, Xiangya Hospital, Central South University, No. 87 Xiangya Road, Kaifu District, Changsha 410008, Hunan, China. ${ }^{2}$ National Clinical Research Center for Geriatric Disorders, Xiangya Hospital, Central South University, No. 87 Xiangya Road, Kaifu District, Changsha 410008, Hunan, China. ${ }^{3}$ Department of Nephrology, Hunan Provincial People's Hospital, The first-affiliated hospital of Hunan normal university, No. 61 Jie-fang West Road, Fu-Rong District, Changsha 410005, Hunan, China.

Received: 23 May 2019 Accepted: 26 February 2020

Published online: 13 March 2020

\section{References}

1. Wyatt RJ, Julian BA. IgA nephropathy. N Engl J Med. 2013;368(25):2402-14. https://doi.org/10.1056/NEJMra1206793.

2. McGrogan A, Franssen CFM, de Vries CS. The incidence of primary glomerulonephritis worldwide: a systematic review of the literature. Nephrol Dial Transplant. 2011;26(2):414-30. https://doi.org/10.1093/ndt/gfa665.

3. De Zhou F, Zhao MH, Zou WZ, Liu G, Wang $H$. The changing spectrum of primary glomerular diseases within 15 years: a survey of 3331 patients in a single Chinese Centre. Nephrol Dial Transplant. 2009;24(3):870-6. https://doi. org/10.1093/ndt/gfn554.

4. Yu H-H, Chiang B-L. Diagnosis and classification of IgA nephropathy. Autoimmun Rev 2014;13(4):556-559. doi:https://doi.org/https://doi.org/10. 1016/j.autrev.2014.01.030

5. Huang C, Li X, Wu J, et al. The landscape and diagnostic potential of T and B cell repertoire in immunoglobulin a nephropathy. J Autoimmun 2019;97: 100-107. doi:https://doi.org/https://doi.org/10.1016/j.jaut.2018.10.018.

6. D'Amico G. Natural history of idiopathic IgA nephropathy: role of clinical and histological prognostic factors. Am J Kidney Dis. 2000;36(2):227-37. https://doi.org/10.1053/ajkd.2000.8966.

7. Lai KN, Tang SCW, Schena FP, et al. IgA nephropathy. Nat Rev Dis Prim 2016:2:16001. https://doi.org/https://doi.org/10.1038/nrdp.2016.1.

8. Nakajima A, Vogelzang A, Maruya $M$, et al. IgA regulates the composition and metabolic function of gut microbiota by promoting symbiosis between bacteria. J Exp Med 2018;215(8):2019-2034. http://dx.doi.org/https://doi.org/ 10.1084/jem.20180427

9. Catanzaro JR, Strauss JD, Bielecka A, et al. IgA-deficient humans exhibit gut microbiota dysbiosis despite secretion of compensatory IgM. Sci Rep. 2019; 9(1):13574. https://doi.org/10.1038/s41598-019-49923-2.

10. Floege J, Feehally J. The mucosa-kidney axis in IgA nephropathy. Nat Rev Nephrol. 2016:12(3):147-56. https://doi.org/10.1038/nrneph.2015.208.

11. Evenepoel P, Poesen R, Meijers B. The gut-kidney axis. Pediatr Nephrol. 2017:32(11):1-10.

12. Olive C, Allen AC, Harper SJ, Wicks ACB, Feehally J, Falk MC. Expression of the mucosal y $T$ cell receptor $V$ region repertoire in patients with $\lg A$ nephropathy. Kidney Int. 1997;52(4):1047-53. https://doi.org/10.1038/ki.1997. 427.

13. Mccarthy DD, Julie K, Cheryl W, et al. Mice overexpressing BAFF develop a commensal flora-dependent, IgA-associated nephropathy. J Clin Invest. 2011:121(10):3991.

14. Felizardo RJF, Castoldi A, Andrade-Oliveira V, Câmara NOS. The microbiota and chronic kidney diseases: a double-edged sword. Clin Transl Immunol. 2016;5(6):e86. https://doi.org/10.1038/cti.2016.36. 
15. Kiryluk K, Li Y, Scolari F, et al. Discovery of new risk loci for IgA nephropathy implicates genes involved in immunity against intestinal pathogens. Nat Genet. 2014;46(11):1187-96. https://doi.org/10.1038/ng.3118.

16. Shreiner $A B$, Kao JY, Young VB. The gut microbiome in health and in disease. Curr Opin Gastroenterol. 2015;31(1):69-75.

17. Forbes JD, Van Domselaar G, Bernstein CN. The gut microbiota in immunemediated inflammatory diseases. Front Microbiol. 2016;7(undefined):1081. https://doi.org/10.3389/fmicb.2016.01081.

18. Xinyun Q, Mingming Z, Xiaotong Y, Na H, Chenggong Y. Faecalibacterium prausnitzii upregulates regulatory $T$ cells and anti-inflammatory cytokines in treating TNBS-induced colitis. J Crohn S Colitis. 2013;7(11):e558-68.

19. Grosserichter-Wagener $C$, Radjabzadeh $D$, van der Weide $H$, et al. Differences in systemic IgA reactivity and circulating Th subsets in healthy volunteers with specific microbiota enterotypes. Front Immunol. 2019; 10(undefined):341. https://doi.org/10.3389/fimmu.2019.00341.

20. Wortelboer K, Nieuwdorp M, Herrema H. Fecal microbiota transplantation beyond Clostridioides difficile infections. EBioMedicine. 2019;44:716-29. https://doi.org/10.1016/j.ebiom.2019.05.066.

21. De Angelis M, Montemurno E, Piccolo M, et al. Microbiota and metabolome associated with Immunoglobulin A Nephropathy (IgAN). PLoS One. 2014; 9(6):e99006. https://doi.org/10.1371/journal.pone.0099006.

22. Escobar JS, Klotz B, Valdes BE, Agudelo GM. The gut microbiota of Colombians differs from that of Americans, Europeans and Asians. BMC Microbiol. 2014;14:311. https://doi.org/10.1186/s12866-014-0311-6.

23. Lerman J. Study design in clinical research: sample size estimation and power analysis. Can J Anaesth 1996;43(2):184-191. http://dx.doi.org/https:// doi.org/10.1007/BF03011261.

24. Faul F, Erdfelder E, Lang A-G, Buchner A. G*power 3: a flexible statistical power analysis program for the social, behavioral, and biomedical sciences. Behav Res Methods 2007;39(2):175-191. http://dx.doi.org/https://doi.org/10.3758/bf03193146.

25. Xianglei $K$, Yingchun $M$, Jianghua $C$, et al. Evaluation of the chronic kidney disease epidemiology collaboration equation for estimating glomerular filtration rate in the Chinese population. Nephrol Dial Transplant. 2013;28(3):641-51.

26. Aßhauer KP, Wemheuer B, Daniel R, Meinicke P. Tax4Fun: predicting functional profiles from metagenomic $16 S$ rRNA data. Bioinformatics. 2015; 31(17):2882-4. https://doi.org/10.1093/bioinformatics/btv287.

27. Collin P, Syrjänen J, Partanen J, Pasternack A, Kaukinen K, Mustonen J. Celiac disease and HLA DQ in patients with IgA nephropathy. Am J Gastroenterol. 2002;97(10):2572-6.

28. Adina W, Birgitta S, Michael F, Ludvigsson JF. Increased risk of IgA nephropathy among individuals with celiac disease. J Clin Gastroenterol. 2013;47(8):678-83.

29. Helin H, Mustonen J, Reunala T, Pasternack A. IgA nephropathy associated with celiac disease and dermatitis herpetiformis. Arch Pathol Lab Med. 1983;107(6):324-7.

30. Donaldson GP, Ladinsky MS, Yu KB, et al. Gut microbiota utilize immunoglobulin A for mucosal colonization. Science .2018;360:795-800. http://dx.doi.org/https://doi.org/10.1126/science.aaq0926.

31. Tanya Y, Rey FE, Manary MJ, et al. Human gut microbiome viewed across age and geography. Nature. 2012;486(7402):222-7.

32. Gentile $\mathrm{CL}$, Weir TL. The gut microbiota at the intersection of diet and human health. Science. 2018;362(6416):776-80.

33. Kothari A, Fulcher CA, Weaver DS, et al. The MetaCyc database of metabolic pathways and enzymes and the BioCyc collection of pathway/genome databases. Nucleic Acids Res. 2015;44(D1):D471-80. https:/doi.org/10.1093/nar/gkv1164.

34. Pan $X$, Xue F, Nan $X$, et al. Illumina sequencing approach to characterize thiamine metabolism related Bacteria and the impacts of thiamine supplementation on Ruminal microbiota in dairy cows fed high-grain diets. Front Microbiol. 2017:8:1818. https://doi.org/10.3389/fmicb.2017.01818.

35. Kunisawa J, Sugiura Y, Wake T, et al. Mode of bioenergetic metabolism during $B$ cell differentiation in the intestine determines the distinct requirement for vitamin $B_{1}$. Cell Rep. 2015;13(1):122-31. https://doi.org/10. 1016/j.celrep.2015.08.063.

36. Kunisawa J. Metabolic changes during B cell differentiation for the production of intestinal lgA antibody. Cell Mol Life Sci. 2017;74(8):1503-9. https://doi.org/10.1007/s00018-016-2414-8.

37. Huggan PJ, Murdoch DR. Fusobacterial infections: clinical spectrum and incidence of invasive disease. J Inf Secur. 2008;57(4):283-9. https://doi.org/ 10.1016/j.jinf.2008.07.016.

38. Manson McGuire A, Cochrane K, Griggs AD, et al. Evolution of invasion in a diverse set of Fusobacterium species. MBio. 2014;5(6):e01864. https://doi. org/10.1128/mBio.01864-14.
39. Shin N-R, Whon TW, Bae J-W. <em>Proteobacteria</em>: microbial signature of dysbiosis in gut microbiota. Trends Biotechnol. 2015;33(9):496503. https://doi.org/10.1016/j.tibtech.2015.06.011.

40. Lupp C, Robertson ML, Wickham ME, et al. Host-mediated inflammation disrupts the intestinal microbiota and promotes the overgrowth of Enterobacteriaceae. Cell Host Microbe. 2007;2(3):204. https://doi.org/10. 1016/j.chom.2007.08.002.

41. Xu K-Y, Xia G-H, Lu J-Q, et al. Impaired renal function and dysbiosis of gut microbiota contribute to increased trimethylamine-N-oxide in chronic kidney disease patients. Sci Rep. 2017;7(1):1445. https://doi.org/10.1038/ s41598-017-01387-y.

42. Wang $F$, Jiang $H$, Shi $K$, Ren $Y$ I, Zhang P, Cheng S. Gut bacterial translocation is associated with microinflammation in end-stage renal disease patients. Nephrology. 2012;17(8):733-8. https://doi.org/10.1111/j.1440-1797.2012.01647.x.

43. Zhang J, Guo Z, Xue Z, et al. A phylo-functional core of gut microbiota in healthy young Chinese cohorts across lifestyles, geography and ethnicities. Isme J 2015;9:1979. https://doi.org/https://doi.org/10.1038/ismej.2015.11.

44. Weiss GA, Chassard C, Hennet T. Selective proliferation of intestinal Barnesiella under fucosyllactose supplementation in mice. Br J Nutr. 2014; 111 (9):1602-10. https://doi.org/10.1017/S0007114513004200.

45. Gomez A, Luckey D, Yeoman CJ, et al. Loss of sex and age driven differences in the gut microbiome characterize arthritis-susceptible 0401 mice but not arthritis-resistant 0402 mice. PLoS One. 2012;7(4):e36095. https://doi.org/10.1371/journal.pone.0036095.

46. Wylie KM, Truty RM, Sharpton TJ, et al. Novel bacterial taxa in the human microbiome. PLoS One. 2012;7(6):e35294.

47. Mancabelli L, Milani C, Lugli GA, Turroni F, Ventura M. Identification of universal gut microbial biomarkers of common human intestinal diseases by meta-analysis. Fems Microbiol Ecol. 2017;93(12):fix153. https://doi.org/10. 1093/femsec/fix153.

48. Sakamoto M, Takagaki A, Matsumoto K, Kato Y, Goto K, Benno Y. Butyricimonas synergistica gen. Nov., sp. nov. and Butyricimonas virosa sp. nov., butyric acid-producing bacteria in the family 'Porphyromonadaceae' isolated from rat faeces. Int J Syst Evol Microbiol. 2009;59(7):1748-53 https:// ijs.microbiologyresearch.org/content/journal/ijsem/10.1099/ijs.0.007674-0.

49. Wong J, Piceno YM, DeSantis TZ, Pahl M, Andersen GL, Vaziri ND. Expansion of urease- and Uricase-containing, Indole- and p-cresol-forming and contraction of short-chain fatty acid-producing intestinal microbiota in ESRD. Am J Nephrol. 2014;39(3):230-7. https://doi.org/10.1159/000360010.

50. Vaziri ND, Wong J, Pahl M, et al. Chronic kidney disease alters intestinal microbial flora. Kidney Int. 2013;83(2):308-15.

51. Schulthess J, Pandey S, Capitani M, et al. The Short Chain Fatty Acid Butyrate Imprints an Antimicrobial Program in Macrophages. Immunity. 2019;50(2):432-45.e7. https://doi.org/10.1016/j.immuni.2018.12.018.

52. Forbes JD, Chen C-Y, Knox NC, et al. A comparative study of the gut microbiota in immune-mediated inflammatory diseases-does a common dysbiosis exist? Microbiome. 2018:6(1):221. https://doi.org/10.1186/s40168-018-0603-4.

53. Qin J, Li Y, Cai Z, et al. A metagenome-wide association study of gut microbiota in type 2 diabetes. Nature. 2012;490:55-60. http://dx.doi.org/ https://doi.org/10.1038/nature11450.

54. Chen J, Wright K, Davis JM, et al. An expansion of rare lineage intestinal microbes characterizes rheumatoid arthritis. Genome Med 2016;8(1):43. http://dx.doi.org/https://doi.org/10.1186/s13073-016-0299-7.

55. Thota VR, Dacha S, Natarajan A, Nerad J. Eggerthella lenta bacteremia in a Crohn's disease patient after ileocecal resection. Future Microbiol 2011;6(5): 595-597. http://dx.doi.org/https://doi.org/10.2217/fmb.11.31.

56. Morales P, Fujio S, Navarrete $P$, et al. Impact of dietary lipids on colonic function and microbiota: an experimental approach involving Orlistatinduced fat Malabsorption in human volunteers. Clin Transl Gastroenterol. 2016;7(4):e161.

57. Jiang $S$, Xie $S, L v D$, et al. Alteration of the gut microbiota in Chinese population with chronic kidney disease. Sci Rep. 2017;7(1):2870. https://doi. org/10.1038/s41598-017-02989-2.

58. Mir RA, Kudva IT. Antibiotic-resistant Shiga toxin-producing Escherichia coli: an overview of prevalence and intervention strategies. Zoonoses Public Health. 2019;66(1):1-13. https://doi.org/10.1111/zph.12533.

59. Rostoker $\mathrm{G}$, Wirquin $\mathrm{V}$, Terzidis $\mathrm{H}$, et al. Mucosal immunity in primary glomerulonephritis. Nephron. 1993:63(3):286-90. https://doi.org/10.1159/ 000187211.

60. Chassaing B, Koren O, Carvalho FA, Ley RE, Gewirtz AT. AlEC pathobiont instigates chronic colitis in susceptible hosts by altering microbiota 
composition. Gut. 2014;63(7):1069-80. https://doi.org/10.1136/gutjpl-2013304909.

61. Flannigan KL, Taylor MR, Pereira SK, et al. An intact microbiota is required for the gastrointestinal toxicity of the immunosuppressant mycophenolate mofetil. J Hear Lung Transplant. 2018;37(9):1047-59. https://doi.org/10.1016/ j.healun.2018.05.002

62. Liu P, Lassén E, Nair V, et al. Transcriptomic and proteomic profiling provides insight into Mesangial cell function in IgA nephropathy. J Am Soc Nephrol. 2017;28(10):2961-72. https://doi.org/10.1681/ASN.2016101103.

63. Fan JM, Liu XR, Zhong X, Ma XY, Zhang YJ, Qin W. External suppression causes the low expression of the Cosmc gene in IgA nephropathy. Nephrol Dial Transplant. 2008;23(5):1608-14. https://doi.org/10.1093/ndt/gfm781.

64. Coppo R, Camilla R, Amore A, et al. Toll-like receptor 4 expression is increased in circulating mononuclear cells of patients with immunoglobulin a nephropathy. Clin Exp Immunol. 2010;159(1):73-81. https://doi.org/10. 1111/j.1365-2249.2009.04045.x.

65. Donadio ME, Loiacono E, Peruzzi L, et al. Toll-like receptors, immunoproteasome and regulatory T cells in children with Henoch-Schönlein purpura and primary lgA nephropathy. Pediatr Nephrol. 2014; 29(9):1545-51. https://doi.org/10.1007/s00467-014-2807-6.

66. Meijers B, Jouret F, Evenepoel P. Linking gut microbiota to cardiovascular disease and hypertension: Lessons from chronic kidney disease. Pharmacol Res. 2018;133(undefined):101-7. https://doi.org/10.1016/j.phrs.2018.04.023.

\section{Publisher's Note}

Springer Nature remains neutral with regard to jurisdictional claims in published maps and institutional affiliations.

Ready to submit your research? Choose BMC and benefit from:

- fast, convenient online submission

- thorough peer review by experienced researchers in your field

- rapid publication on acceptance

- support for research data, including large and complex data types

- gold Open Access which fosters wider collaboration and increased citations

- maximum visibility for your research: over $100 \mathrm{M}$ website views per year

At $\mathrm{BMC}$, research is always in progress.

Learn more biomedcentral.com/submissions 